\title{
Disorder trapping by rapidly moving phase interface in an undercooled liquid
}

\author{
Peter Galenko ${ }^{1, *}$, Denis Danilov ${ }^{2}$, Irina Nizovtseva ${ }^{1,3}$, Klemens Reuther ${ }^{1}$, and Markus \\ Rettenmayr ${ }^{1}$ \\ ${ }^{1}$ Friedrich-Schiller-Universität Jena, Physikalisch-Astronomische Fakultät, D-07743 Jena, Germany \\ ${ }^{2}$ Institute of Nanotechnology, Karlsruhe Institute of Technology, 76344 Eggenstein-Leopoldshafen, \\ Germany \\ ${ }^{3}$ Ural Federal University, Laboratory of Multi-Scale Mathematical Modeling, 620000 Ekaterinburg, \\ Russian Federation
}

\begin{abstract}
Non-equilibrium phenomena such as the disappearance of solute drag, the origin of solute trapping and evolution of disorder trapping occur during fast transformations with originating metastable phases [D.M. Herlach, P.K. Galenko, D. Holland-Moritz, Metastable solids from undrercooled melts (Elsevier, Amsterdam, 2007)]. In the present work, a theoretical investigation of disorder trapping by a rapidly moving phase interface is presented. Using a model of fast phase transformations, a system of governing equations for the diffusion of atoms, and the evolution of both long-range order parameter and phase field variable is formulated. First numerical solutions are carried out for a congruently melting binary alloy system.
\end{abstract}

\section{Introduction}

Disorder trapping is a known non-equilibrium phenomenon occurring during transformations of congruently melting ordered intermetallic compounds [1,2]. This phenomenon occurs especially during rapid crystallization of undercooled melts of intermetallics with a superlattice crystalline structure [3-5]. In such systems, the phase interface propagation is sluggish at small undercoolings. The attachment of atoms from the liquid to the phase interface needs short-range atomic diffusion, since atoms have to move to the proper lattice site in the superlattice structure. If the undercooling increases, the non-equilibrium effect of disorder trapping leads to the formation of a metastable disordered structure. Experimental evidence of disorder trapping has been demonstrated by in situ diffraction studies using synchrotron radiation on levitation-processed samples, in which a transition from ordered to disordered growth at a critical undercooling was unambiguously shown [6]. The phenomenon of order-disorder transition with distinct change of the order parameter is also important in structural transformations with non-monotonic relaxation processes of melts [8] and in liquid-liquid phase separation of undercooled metallic alloys $[9,10]$.

The disorder trapping was investigated from theoretic perspective by sharp interface models $[2,3,6]$, a diffuse interface model [11] and methods of atomistic simulation [1, 12]. In the present work, using an existing approach for fast phase transformations [13], a diffuse

\footnotetext{
*e-mail: peter.galenko@uni-jena.de
} 
interface model results in a system of hyperbolic equations to describe (i) the rapid interface motion and (ii) the transition from ordered to disordered structures formed from undercooled liquids. The pioneering atomistic model of Chernov [1] and thermodynamic model of Boettinger and Aziz [2] consider a process of ordering at the interface only. The present diffuse interface model introduces spatial variation of order parameter such that the order-disorder transition and relaxation of order parameter exist in the diffuse zone between phases as well as in a solid phase far behind the solid-liquid interface. In addition to previous works [1$3,6,11]$, the present model takes into account highly rapid interface propagation at which locally non-equilibrium states in chemical composition and in atomic ordering are freezing in solid phase with an appearance of metastable states.

In the numerical solutions of the model, we test the existence of pronounced disorder trapping by direct exchange of dissimilar atoms between sublattices at high interface velocities moving in an undercooled binary liquid. The main focus of the present work is to analyze numeric results and to formulate conditions for disorder trapping in rapidly transforming congruently melting alloy undercooled in the liquid state.

\section{Definitions}

\subsection{Main functions}

We consider a binary mixture consisting of A-atoms and B-atoms. For a given temperature $T$, the main functions of the model, which should be obtained by solutions, are defined as follows. The first function is $\varphi$ the phase field with $\varphi=1$ in the $S$-phase (solid) and $\varphi=0$ in the $L$-phase (liquid). The second function is $x_{B}$ the overall continuous concentration of B-atoms (which can be defined through the $L$-phase and $S$-phase concentrations). Concentrations in the $S$-phase are given by $x_{i}^{j}=n_{i}^{j} /\left(n_{A}^{j}+n_{B}^{j}\right)$, where $n_{i}^{j}$ is the number of moles of atom $i(i=\mathrm{A}, \mathrm{B})$ on the sublattice $j(j=\alpha, \beta)$. Finally, the third function is the long-range order parameter $\eta$, which is defined by the concentrations $x_{i}^{j}=\left(x_{A}^{\alpha}, x_{A}^{\beta}, x_{B}^{\alpha}, x_{B}^{\beta}\right)$ of the $\alpha-$ and $\beta$-sublattices as

$$
\eta=x_{A}^{\alpha}-x_{A}^{\beta}=x_{B}^{\beta}-x_{B}^{\alpha}
$$

A completely disordered state in the $S$-phase occurs with $\eta=0$. Atoms exhibit full order on sublattices if $\eta=1$. The concentrations in the $S$-phase on sublattices are

$$
\begin{aligned}
x_{A}^{\alpha} & =1-x_{B}-\frac{\eta}{2}, & x_{B}^{\alpha} & =x_{B}-\frac{\eta}{2}, \\
x_{A}^{\beta} & =1-x_{B}+\frac{\eta}{2}, & x_{B}^{\beta} & =x_{B}+\frac{\eta}{2} .
\end{aligned}
$$

\subsection{Gibbs free energy}

\subsubsection{The entire system}

The Gibbs free energy for the entire binary system is given by

$$
\begin{aligned}
G\left(\varphi, \nabla \varphi, \eta, \nabla \eta, x_{B}\right)=G_{S}\left(x_{B}, \eta\right) p(\varphi)+ & G_{L}\left(x_{B}\right) p(1-\varphi) \\
& +\frac{\varepsilon_{\varphi}}{2}(\nabla \varphi)^{2}+\frac{\varepsilon_{\eta}}{2}(\nabla \eta)^{2}+W_{\varphi} g_{\varphi}(\varphi)+W_{\eta} g_{\eta}(\eta) .
\end{aligned}
$$

The double-well functions in Eq. (4) for the phase field $g_{\varphi}$ and for the long-range order parameter $g_{\eta}$ are given by

$$
g_{\varphi}(\varphi)=\varphi^{2}(1-\varphi)^{2}, \quad g_{\eta}(\eta)=\eta^{2}(1-\eta)^{2} .
$$


The Gibbs free energy is interpolated between the phases using the following function

$$
p(\varphi)=\varphi^{2}(3-2 \varphi)
$$

\subsubsection{The phases}

In the $L$-phase we assume the following approximation

$$
\begin{gathered}
G_{L}\left(T, x_{B}\right)=\left(1-x_{B}\right) G_{L}^{A}(T)+x_{B} G_{L}^{B}(T), \\
G_{L}^{A}\left(T, x_{B}\right)=G_{L}^{0 A}(T)+R T \ln \left(1-x_{B}\right)+x_{B} \Omega_{L}, \\
G_{L}^{B}\left(T, x_{B}\right)=G_{L}^{0 B}(T)+R T \ln \left(x_{B}\right)+\left(1-x_{B}\right) \Omega_{L},
\end{gathered}
$$

which can be considered as contribution (7) from elements $A$ and $B$, reference terms $G_{L}^{0 i}$, ideal mixture terms $R T \ln x_{i}$, and excess terms $x_{i} \Omega_{L}$ (where again $i=A, B$ ).

In the $S$-phase, the Gibbs free energy has additional terms related to the disorder parameter

$$
G_{S}\left(T, x_{B}, \eta\right)=G_{S}^{r e f}\left(T, x_{B}\right)+G_{S}^{i d}\left(T, x_{B}, \eta\right)+G_{S}^{e x}\left(x_{B}, \eta\right) .
$$

Assuming that the $L$-phase transforms into the body centered cubic modification of the $S$ phase, the following contributions into the free energy (10) are taken into account:

- reference contribution

$$
G_{S}^{r e f}\left(T, x_{B}\right)=\left(1-x_{B}\right) G_{A}^{b c c}(T)+x_{B} G_{B}^{b c c}(T),
$$

- ideal mixture contribution

$$
\begin{aligned}
G_{S}^{i d}\left(T, x_{B}, \eta\right)=\frac{1}{2} R T & \left(\left(x_{B}-\frac{\eta}{2}\right) \ln \left(x_{B}-\frac{\eta}{2}\right)+\left(x_{B}+\frac{\eta}{2}\right) \ln \left(x_{B}+\frac{\eta}{2}\right)\right. \\
& \left.+\left(1-x_{B}-\frac{\eta}{2}\right) \ln \left(1-x_{B}-\frac{\eta}{2}\right)+\left(1-x_{B}+\frac{\eta}{2}\right) \ln \left(1-x_{B}+\frac{\eta}{2}\right)\right)
\end{aligned}
$$

- excess free energy

$$
G_{S}^{e x}\left(x_{B}, \eta\right)=\Omega_{2}\left(x_{B}\left(1-x_{B}\right)+\frac{\eta^{2}}{4}\right)+\Omega_{3} \eta^{3}+\Omega_{4} \eta^{4} .
$$

\section{Governing equations}

Following the model of fast phase transformations [13], one can derive governing equations represented by hyperbolic equations for non-conserved and conserved field variables. They were obtained by the condition of non-decreasing entropy in time that, for isothermal systems, is equivalent to the condition of non-increasing Helmholtz free energy in time [14]. Following these approaches $[13,14]$, one can derive the system of governing equations using the Gibbs free energies from Section 2.2 as thermodynamic potentials accessible from thermodynamic databases. Then, one can find governing equations for

- the phase field

$$
\tau_{\varphi} \frac{\partial^{2} \varphi}{\partial t^{2}}+\frac{\partial \varphi}{\partial t}=-M_{\varphi} \frac{\delta G}{\delta \varphi}
$$

- the long-range order parameter

$$
\tau_{\eta} \frac{\partial^{2} \eta}{\partial t^{2}}+\frac{\partial \eta}{\partial t}=-M_{\eta} \frac{\delta G}{\delta \eta}
$$


- the concentration and chemical potential

$$
\tau_{D} \frac{\partial^{2} x_{B}}{\partial t^{2}}+\frac{\partial x_{B}}{\partial t}=\nabla \cdot\left(M_{x} \nabla \mu_{B}\right), \quad \mu_{B}=\frac{\delta G}{\delta x_{B}} .
$$

The hyperbolic equations (14) and (15) are damped wave equations, which extend known parabolic equation for the non-conserved order parameter derived first by Mandel'shtamLeontovich $[15,16]$ and known in literature as the time-dependent Ginzburg-Landau equation [16, 17] or Allen-Cahn equation [18]. The hyperbolic equation (16) generalizes the known parabolic equation for the conserved order parameter derived and analyzed first by Khachaturyan [19] and Cahn and Hilliard [20]. Equations (14)-(16) were applied to nonmonotonic relaxation [7, 8], phase segregation [9] and were analyzed in the context of fast dynamics during transitions from un(meta)stable to (meta)stable states [21]. The validity of hyperbolic type models in fast phase transition theory has been verified by comparison with experimental data [22], in molecular dynamics simulations of the solute trapping effect by rapidly moving interfaces [23] and by coarse graining derivations of phase field equations [24].

\subsection{Phase-field}

In its explicit form, the governing equation for the phase field comes from Eqs. (4)-(14). As a result one finds

$$
\begin{aligned}
& \frac{\tau_{\varphi}}{M_{\varphi}} \frac{\partial^{2} \varphi}{\partial t^{2}}+\frac{1}{M_{\varphi}} \frac{\partial \varphi}{\partial t}=\varepsilon_{\varphi} \nabla^{2} \varphi-W_{\varphi} \frac{d g_{\varphi}(\varphi)}{d \varphi}-\left[\left(1-x_{B}\right) G_{A}^{b c c}+x G_{B}^{b c c}\right. \\
&+ \frac{R T}{2}\left(\left(x_{B}-\eta / 2\right) \ln \left(x_{B}-\eta / 2\right)+\left(x_{B}+\eta / 2\right) \ln \left(x_{B}+\eta / 2\right)\right. \\
&\left.+\left(1-x_{B}-\eta / 2\right) \ln \left(1-x_{B}-\eta / 2\right)+\left(1-x_{B}+\eta / 2\right) \ln \left(1-x_{B}+\eta / 2\right)\right) \\
&\left.+\Omega_{2}\left(x_{B}\left(1-x_{B}\right)+(1 / 4) \eta^{2}\right)+\Omega_{3} \eta^{3}+\Omega_{4} \eta^{4}\right] \frac{d p(\varphi)}{d \varphi} \\
&-\left(\left(1-x_{B}\right)\left(G_{L}^{0 A}+R T \ln \left(1-x_{B}\right)+x_{B} \Omega_{L}\right)+x_{B}\left(G_{L}^{0 B}+R T \ln \left(x_{B}\right)+\left(1-x_{B}\right) \Omega_{L}\right)\right) \frac{d p(\varphi)}{d \varphi}
\end{aligned}
$$

Without the ordering process, i.e. if $\eta=0$, Eq. (17) transforms into the one derived in Refs. [13, 14].

\subsection{Order parameter}

Atomic ordering in $\alpha$ - and $\beta$-sublattices is described by Eq. (15) together with the free energy (4)-(13) by the following equation:

$$
\begin{array}{r}
\frac{\tau_{\eta}}{M_{\eta}} \frac{\partial^{2} \eta}{\partial t^{2}}+\frac{1}{M_{\eta}} \frac{\partial \eta}{\partial t}=\varepsilon_{\eta} \nabla^{2} \eta-W_{\eta} \frac{d g_{\eta}(\eta)}{d \eta}-\left[\frac { R T } { 2 } \left(-\frac{1}{2} \ln \left(x_{B}-\eta / 2\right)+\frac{1}{2} \ln \left(x_{B}+\eta / 2\right)\right.\right. \\
\left.-\frac{1}{2} \ln \left(1-x_{B}-\eta / 2\right)+\frac{1}{2} \ln \left(1-x_{B}+\eta / 2\right)\right) \\
\left.+\frac{1}{2} \Omega_{2} \eta+3 \Omega_{3} \eta^{2}+4 \Omega_{4} \eta^{3}\right] p(\varphi)
\end{array}
$$


From Eq. (18) it follows that the process of atomic ordering is coupled with the phase field by the interpolation function $p(\varphi)$.

\subsection{Chemical diffusion}

The chemical potential from Eq. (16) is given by the variational derivative

$$
\begin{aligned}
& \mu_{B}=\frac{\delta G}{\delta x_{B}}= \\
& \qquad \begin{aligned}
G_{B}^{b c c}-G_{A}^{b c c}+\frac{R T}{2}( & \left.\left.\ln \left(x_{B}-\frac{\eta}{2}\right)+\ln \left(x_{B}+\frac{\eta}{2}\right)-\ln \left(1-x_{B}-\frac{\eta}{2}\right)-\ln \left(1-x_{B}+\frac{\eta}{2}\right)\right)+\Omega_{2}\left(1-2 x_{B}\right)\right] p(\varphi) \\
& +\left[G_{L}^{0 B}-G_{L}^{0 A}+R T\left\{\ln \left(x_{B}\right)-\ln \left(1-x_{B}\right)\right\}+2\left(1-2 x_{B}\right) \Omega_{L}\right] p(1-\varphi)
\end{aligned}
\end{aligned}
$$

The mobility $M_{x}$ in the diffusion equation Eq. (16) is an interpolation between the bulk mobilities in the liquid $M_{L}$ and in the solid $M_{S}$ :

$$
M_{x}=M_{L}(1-p(\varphi))+M_{S}(\eta) p(\varphi)=M_{L}(1-p(\varphi))+\left(M_{S}^{\text {disorder }}(1-\eta)+M_{S}^{\text {order }} \eta\right) p(\varphi) .
$$

As follows from Eq. (20), the bulk mobility $M_{S}(\eta)$ in the solid is interpolated by atomic mobilities $M_{S}^{\text {order }}$ and $M_{S}^{\text {disorder }}$ in the ordered state and disordered state, respectively.

Table 1. Physical parameters of the $\mathrm{A}_{50} \mathrm{~B}_{50}$ alloy used for phase field modeling

\begin{tabular}{ccc}
\hline Parameter & Value & Reference \\
\hline Concentration of A(B), $x_{B}$ & 0.5 mole fraction & present work \\
$\eta$-rate relaxation time, $\tau_{\eta}$ & $4 \cdot 10^{-8} \mathrm{~s}$ & present work \\
$\varphi$-rate relaxation time, $\tau_{\varphi}$ & $4 \cdot 10^{-11} \mathrm{~s}$ & present work \\
$x_{B}$-rate relaxation time, $\tau_{D}$ & $4 \cdot 10^{-10} \mathrm{~s}$ & present work \\
Mobility of the $\eta$-field, $M_{\eta}$ & $8 \cdot 10^{4} \mathrm{~mole} \cdot \mathrm{J}^{-1} \cdot \mathrm{s}^{-1}$ & present work \\
Mobility of the $\varphi$-field, $M_{\varphi}$ & $4 \cdot 10^{5} \mathrm{~mole} \cdot \mathrm{J}^{-1} \cdot \mathrm{s}^{-1}$ & present work \\
Mobility of B-atoms in the $L$-phase, $M_{L}$ & $2 \cdot 10^{-8} \mathrm{~mole} \cdot \mathrm{m}^{2} \cdot \mathrm{J}^{-1} \cdot \mathrm{s}^{-1}$ & present work \\
Mobility of B-atoms in the disordered state, $M_{S}^{\text {disorder }}$ & $7 \cdot 10^{-11} \mathrm{~mole} \cdot \mathrm{m}^{2} \cdot \mathrm{J}^{-1} \cdot \mathrm{s}^{-1}$ & present work \\
Mobility of B-atoms in the ordered state, $M_{S}^{\text {order }}$ & $9 \cdot 10^{-12} \mathrm{~mole} \cdot \mathrm{m}^{2} \cdot \mathrm{J}^{-1} \cdot \mathrm{s}^{-1}$ & present work \\
Gradient factor for the $\eta$-field, $\varepsilon_{\eta}$ & $1.4 \cdot 10^{-13} \mathrm{~J} \cdot \mathrm{m}^{2} \cdot \mathrm{mole}^{-1}$ & present work \\
Gradient factor for the $\varphi$-field, $\varepsilon_{\varphi}$ & $5.5 \cdot 10^{-12} \mathrm{~J} \cdot \mathrm{m}^{2} \cdot \mathrm{mole}^{-1}$ & present work \\
Energy barrier between states in the $\eta$-field, $W_{\eta}$ & $2.5 \cdot 10^{3} \mathrm{~J} \cdot \mathrm{mole}^{-1}$ & present work \\
Energy barrier between states in the $\varphi$-phase, $W_{\varphi}$ & $4.5 \cdot 10^{3} \mathrm{~J} \cdot \mathrm{mole}^{-1}$ & present work \\
1st Thermodynamic parameter, $\Omega_{2}$ & $-2 \cdot 10^{3} \mathrm{R} \mathrm{J/mole}$ & [2] \\
2nd Thermodynamic parameter, $\Omega_{3}$ & $-1.225 \cdot 10^{6} \mathrm{R} \mathrm{J} / \mathrm{mole}$ & present work \\
3rd Thermodynamic parameter, $\Omega_{4}$ & $-5.662 \cdot 10^{2} \mathrm{R} \mathrm{J/mole}$ & [2] \\
4th Thermodynamic parameter, $\Omega_{L}$ & $-1.72 \cdot 10^{4} \mathrm{~J} / \mathrm{mole}$ & [2] \\
\hline
\end{tabular}

\section{Material parameters and modeling}

The spatially inhomogeneous evolution of ordered and disordered states has been modeled by the numerical solution of the equations for diffusion (16), (19) and (20), phase field motion (17) and ordering (18). Material parameters have been chosen for a binary congruently melting $\mathrm{A}_{50} \mathrm{~B}_{50}$ alloy. Functions $G_{L}^{0 A}(T), G_{L}^{0 A}(T), G_{A}^{b c c}(T)$, and $G_{B}^{b c c}(T)$ from Eqs. (7)-(9) and 


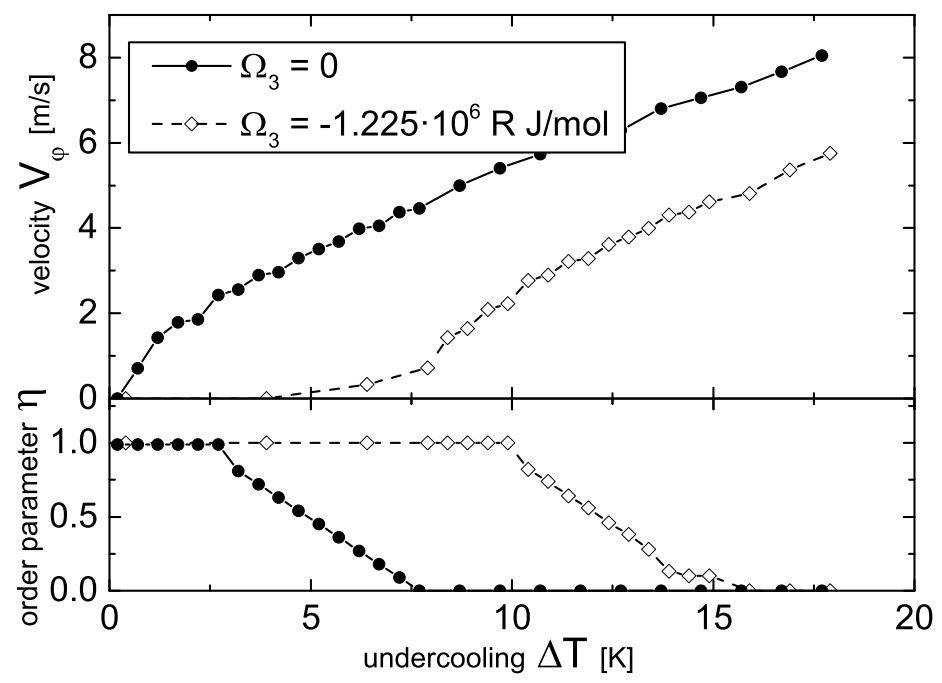

Figure 1. Behavior of the phase field interface velocity $V_{\varphi}$ and long-range order parameter $\eta$ as functions of liquid undercooling $\Delta T=T_{L}-T$, where $T_{L}$ is the liquidus temperature (calculated as the temperature for "liquid-ordered phase" co-existence). Two cases show the change of the transformation kinetics depending on the thermodynamic parameter $\Omega_{3}$ which regulates the barrier height for atomic diffusion jumps between sublattices $\alpha$ and $\beta$, see Eq. (13).

(11) are taken from the thermodynamical data base of elements (CALPHAD) for a $\mathrm{Ni}_{50} \mathrm{Al}_{50}$ alloy.

The numerical scheme for the solution of the model consists of a finite difference (FD) scheme of second order with explicit time stepping. The initial concentration distribution was taken as homogeneous, $x_{B}=0.5$, while both phase field $\varphi$ and order parameter $\eta$ were initialized to unity in the solid and zero in the liquid, connected by a smooth transition at the interface, which was described by a Gaussian error function with a width of 1 micron, corresponding to 10 FD nodes. Boundary conditions were Dirichlet type conditions on the solidifying side and Neumann type (i.e., zero flux) conditions in the liquid far away from the interface.

Numerical solutions of Eqs. (16), (19), (20), 17), and (18) were achieved in a onedimensional space. To obtain steady state results, average values of the interface velocity $V_{\varphi}$ for the phase field and the long-range order parameter $\eta$ where determined as follows. The phase-field has a step-like shape with $\varphi=1$ in the solid and $\varphi=0$ in the liquid. Because the solid-liquid interface is a diffuse interface, the conventional definition of the interface position is the point $z_{f}$ where $\varphi\left(z_{f}\right)=0.5$. In this work we adopt another definition of the interface position. $z_{f}=\int_{-\infty}^{+\infty} \varphi\left(z^{\prime}\right) d z^{\prime}$ is exact for the case of a symmetric interface profile and allows us to find an exact interface velocity in case of a steady state profile. During the simulation run, we collect the values of the interface position $z_{f}^{n}$ at times $t^{n}$ after a fixed number (e.g. 2000) of time steps. The current interface velocity $V_{\varphi}$ is obtained as $V_{\varphi}^{n}=\left(z_{f}^{n}-z_{f}^{n-1}\right) /\left(t^{n}-t^{n-1}\right)$. After the system reaches a steady state regime, we calculate the steady state interface velocity $V_{\varphi}^{(a v g)}$ as the average of the last ten values $V_{\varphi}^{n}$. The average value of the order parameter $\eta$ in the solid phase right beyond the interface is determined as the average of 11 FD nodes 


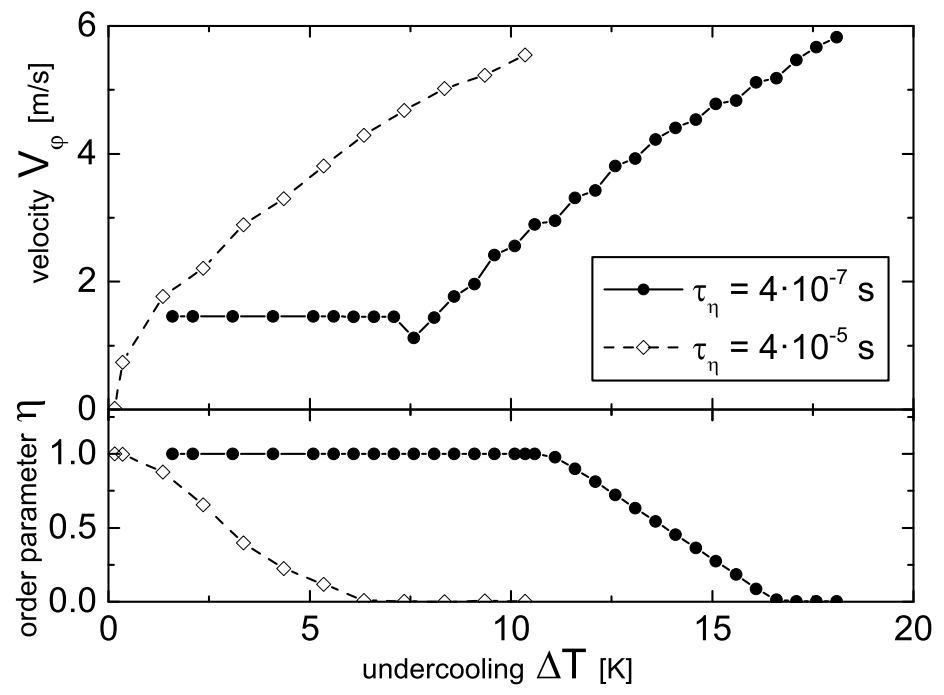

Figure 2. Behavior of the phase field interface velocity $V_{\varphi}$ and long-range order parameter $\eta$ as functions of liquid undercooling $\Delta T=T_{L}-T$, where $T_{L}$ is the liquidus temperature (calculated as the temperature for "liquid-ordered phase" co-existence). Two cases show the change of the transformation kinetics depending of the time $\tau_{\eta}$ for relaxation of the ordering rate $\partial \eta / \partial t$ to change the regime from inertial (ballistic) to dissipative (diffusive) [21]. The $\eta$-gradient factor has been chosen as $\varepsilon_{\eta}=1.4 \cdot 10^{-11}\left(\mathrm{~J} \cdot \mathrm{m}^{2} \cdot \mathrm{mole}^{-1}\right)$.

at a distance of 10 FD spacings behind the interface, i.e. the FD nodes 10 to 20 behind the calculated interface position.

\section{Results}

Figures 1 and 2 show the influence of various parameters for the ordering process on both the transition from $L$-phase to $S$-phase and on the ordering process itself. Namely, Fig. 1 presents the kinetics change due to different values of $\Omega_{3}$. It can be seen that with a non-zero value of the barrier $\Omega_{3}$ [see Eq. (13)] the kinetics of transformation qualitatively changes with a decrease in $V_{\varphi}$ and a shift in the disorder transformation velocity $V_{D T}$ to its large values. Figure 2 illustrates the influence of gradient factor and relaxation time $\tau_{\eta}$ on the transformation kinetics. The time $\tau_{\eta}$ characterizes the relaxation to local equilibrium in the $\eta$-field, i.e., the relaxation of the rate $\partial \eta / \partial t$. An increase in the relaxation time $\tau_{\eta}$ moves the initiation of disorder trapping to smaller undercoolings.

\section{Kinetics and temporal conditions for trapping of disorder}

Finally, on the basis of our numerical results (see Section 5), we formulate a condition of disorder trapping by a moving diffuse interface. During fast transformations the disorder trapping effect occurs when a particular atom has no time to find its equilibrium position within the diffuse interface and remains in a higher energetic position. Instead of a phase with complete atomic order, a disordered metastable phase is appearing behind the diffuse interface. This effect becomes important at phase interface velocities $V_{\varphi}$ larger than a characteristic 


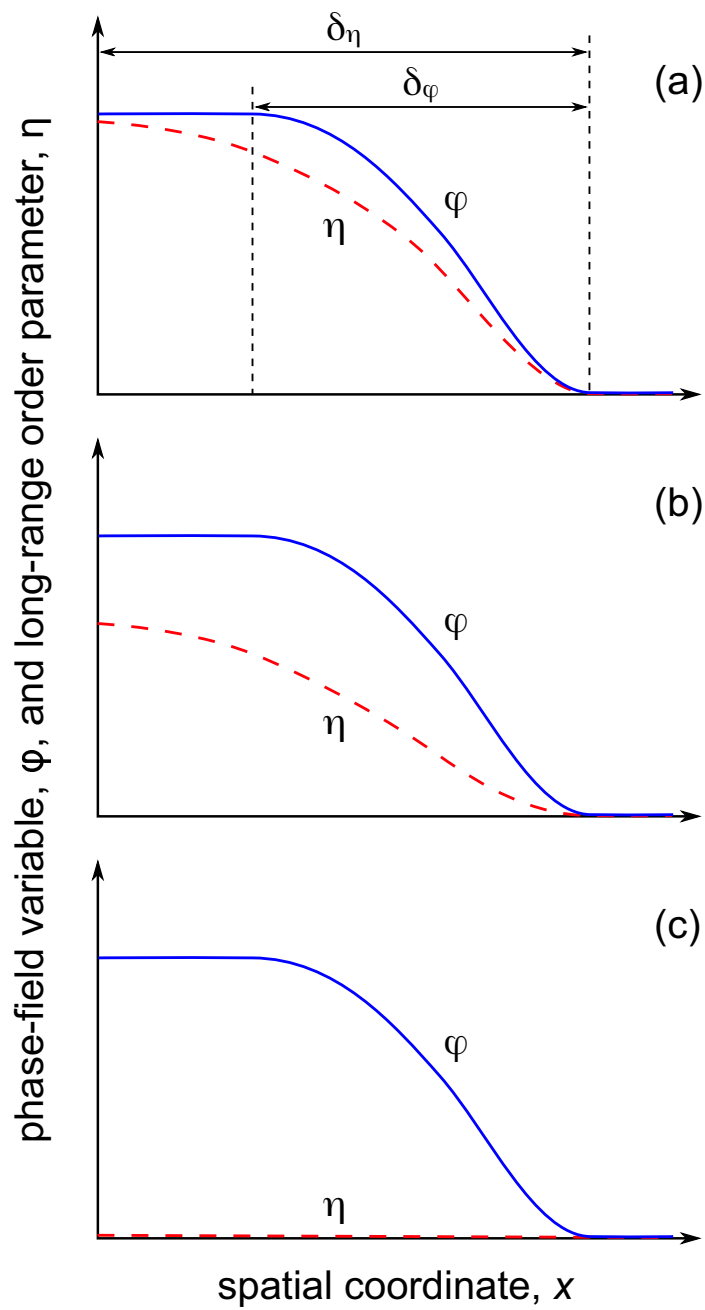

Figure 3. Profiles of the phase field $\phi$ and long-range order parameter $\eta$ with their thickness $\delta_{\varphi}$ and $\delta_{\eta}$, respectively. (a) Fully ordered state, $V_{\varphi} \ll V_{D T}$. (b) Partial disorder trapping, $V_{\varphi} \approx V_{D T}$. (c) Complete disorder trapping, $V_{\varphi}>V_{D T}$.

value of the order of $V_{D T} \propto D_{\eta} / \delta_{\eta}$ (with $D_{\eta}=M_{\eta} \varepsilon_{\eta}$ being the characteristic diffusion coefficient in solid phase for the ordering process, and $\delta_{\eta}$ the thickness of the interface for ordering). Indeed, when $V_{\varphi}>V_{D T}$, the characteristic time $\delta_{\varphi} / V_{\varphi}$ of the diffuse interface of thickness $\delta_{\varphi}$ becomes smaller than the typical time $\delta_{\eta}^{2} / D_{\eta}$ required for complete ordering within the diffuse interface. As a result, disorder trapping by the diffuse interface exists under the velocity condition $V>D_{\eta} / \delta_{\eta}$, or under the time scale condition $\delta_{\varphi} / V_{\varphi}<\delta_{\eta}^{2} / D_{\eta}=\delta_{\eta} / V_{D T}$. From these conditions it follows that the critical velocity for the disorder trapping, $V_{D T}$, becomes smaller as the ratio between the thickness of the ordering zone $\delta_{\eta}$ and the diffuse interface thickness $\delta_{\varphi}$ increases:

$$
V_{D T}<\frac{\delta_{\eta}}{\delta_{\varphi}} V_{\varphi}
$$


As follows from the inequality (21), the disorder trapping becomes more pronounced with the increase of the diffuse interface velocity $V_{\varphi}$ and the decrease of the diffuse interface thickness $\delta_{\varphi}$. Furthermore, with a "sharper" ordering zone, i.e., with decreasing $\delta_{\eta}$, the beginning of disorder trapping is shifted to larger phase interface velocities. These situations are illustrated in Fig. 3.

\section{Conclusions}

1. Using a phase field method in the theory of fast phase transformations, a new model for disorder trapping by a rapidly moving interface is formulated. The model consists of a system of hyperbolic equations for mass transport by atomic diffusion, dynamics of the phase field, and dynamics of atomic ordering.

2. The system of model equations has been solved numerically to reach steady-state from initially non-stationary states. For these steady-states, the interface velocity and long-range order parameter are analyzed depending on the type of free energy and relaxation time $\tau_{\eta}$ for the rate of long-range order parameter $\partial \eta / \partial t$.

3. It has been shown quantitatively that there are critical undercoolings at which the trapping of disorder begins and finishes with the formation of a completely disordered phase. Using the results of our computations, a criterion for disorder trapping by rapid diffuse interface has been formulated.

\section{Acknowledgments}

P. G. specially acknowledges the support the Russian Science Foundation (project no. 1611-10095). I. N. acknowledges the support of Alexander von Humboldt Foundation (ID 1160779) and the Council on grants and scholarships (MK-3124.2015.5, SP-3122.2015.5). M.R. and K.R. acknowledge support from the Deutsche Forschungsgemeinschaft (DFG, grants no. Re 1261/8-2 and Re 1261/18-1).

\section{References}

[1] A.A. Chernov, Sov. Phys. JETP 26, 1182 (1968)

[2] W.J. Boettinger, M.J. Aziz, Acta Metall. 37, 3379 (1989)

[3] M. Barth, B. Wei, D.M. Herlach, Phys. Rev. B 51, 3422 (1995)

[4] R. Ahmad, R.F. Cochrane, A.M. Mullis, J. Mater. Sci. 47, 2411 (2012)

[5] C. Yang, J. Gao, J. Cryst. Growth 394, 24 (2014).

[6] H. Hartmann, D. Holland-Moritz, P.K. Galenko, D.M. Herlach, Europhys. Lett. 87, 40007 (2009)

[7] V.I. Lad'yanov, M.G. Vasin, S.V. Logunov, V.P. Bovin, Phys. Rev. B 62, 12107 (2000)

[8] M.G. Vasin, V.I. Ladianov, Phys. Rev. E 68, 051202 (2003)

[9] P. Galenko, Phys. Lett. A 287, 190 (2001)

[10] E. Davidoff, P.K. Galenko, D.M. Herlach, M. Kolbe, N. Wanderka, Acta Mater. 61, 1078 (2013)

[11] H. Assadi, Acta Mater. 55, 5225 (2007)

[12] X.Q. Zhang, Y. Yang, Y.F. Gao, J.J. Hoyt, M. Asta, D.J. Sun, Phys. Rev. B 85, 041601 (2012)

[13] P. Galenko, D. Jou, Phys. Rev. E 71, 046125 (2005)

[14] V.G. Lebedev, A.V. Sysoeva, P.K. Galenko, Phys. Rev. E 83, 026705 (2011) 
[15] L.I. Mandel'shtam and M.A. Leontovich, JETP 7, 438 (1937)

[16] A.Z. Patashinskii, V.L. Pokrovskii, Fluctuation theory of phase transitions (Pergamon, Oxford, 1979)

[17] E.M. Lifshitz, L.P. Pitaevskii, Physical Kinetics. Course Theoretical Physics, Vol 10, (Pergamon Press, Oxford, UK, 1981)

[18] S.M. Allen, J.W. Cahn, Acta Metall. 27, 1085 (1979)

[19] A.G. Khachaturyan, Theory of Structural Transformations in Solids (Wiley, New York, 1983)

[20] J.W. Cahn, J.E. Hilliard, J. Chem. Phys. 28, 258 (1958)

[21] P. Galenko, D. Danilov, V. Lebedev, Phys. Rev. E 79, 051110 (2009)

[22] P.K. Galenko and D.M. Herlach, Phys. Rev. Lett. 96, 150602 (2006)

[23] Y. Yang, H. Humadi, D. Buta D, B.B. Laird, D. Sun, J.J. Hoyt, M. Asta, Phys. Rev. Lett. 107, 025505 (2011)

[24] D. Jou, P. Galenko, Phys. Rev. E 88, 042151 (2013) 